\title{
Organização dos Prontuários dos Pacientes em uma Unidade Básica de Saúde
}

Organization of Patient Records in a Basic Health Unit

Organization of Patient Records in a Basic Health Unit

\begin{abstract}
Milene Arlinda de Lima Mendes ${ }^{1}$
Elaine Silva dos Santos ${ }^{2}$
\end{abstract}

\section{Resumo}

Objetivo: contribuir para a organização dos prontuários em uma unidade básica de saúde (UBS) de Maceió. Método: para fundamentar o plano de intervenção foram realizadas pesquisas na base de dados da Scientific Electronic Library Online (SciELO), Google Acadêmico com os descritores: prontuários; capacitação em serviço e arquivo. Os documentos da Secretaria Municipal de Saúde de Maceió e UBS também subsidiaram esse trabalho. Resultados Esperados: promover a capacitação dos profissionais da recepção e do arquivo sobre técnicas e métodos arquivistas e legislação que permeia os prontuários dos pacientes; organizar os prontuários dos pacientes na unidade de saúde referenciada e implantar uma planilha eletrônica, facilitando a identificação dos prontuários dos pacientes de maneira mais ágil, auxiliando assim, a rotina dos profissionais. Conclusão: A partir deste estudo verificou-se que os prontuários são documentos em que se registra todos os cuidados realizados pela equipe multiprofissional a cada usuário. Deste modo, se configuram como importantes ferramentas para: subsidiar pesquisas científicas na área da saúde, auxiliar os profissionais no

${ }^{1}$ Mestre em Gestão de Sistemas de e-Learning pela Universidade Nova de Lisboa. Especialista em Gestão do Trabalho e Educação em Saúde UFAL. Endereço completo: Rua Silverio Jorge. N 1174. Jaraguá. 57022-050. Maceió - Al, Brasil. E-mail: milenemendes@gmail.com

${ }^{2}$ Biológa e Especialista em Gestão do Trabalho e Educação em Saúde pela Universidade Federal de Alagoas.

Recebido: Dez/2017 - Aceito: Abr/2018. 
monitoramento e avaliação da saúde de seus pacientes, planejamento das ações de saúde, analise do perfil epidemiológico de cada território, além de servir de prova cabal para demandas externas, como ações judiciais, do conselho de ética e de classes profissionais, acreditação etc. Apesar do exposto, nota-se que o tratamento dado a esses documentos ainda está longe de ser o ideal e a unidade de saúde em foco não refuta essa realidade, logo a proposta aqui apresentada se faz necessária.

\section{Descritores: Registros Médicos; Capacitação em Serviço; Arquivo.}

\section{Abstract}

Objective: to contribute to the organization of medical records in a basic health unit (UBS) in Maceió. Method: to base the intervention plan were carried out searches in the database of the Scientific Electronic Library Online (SciELO), Google Scholar with the descriptors: medical records; training in service and archiving. The documents of the Municipal Health Department of Maceio and UBS also subsidized this work. Expected Outcomes: to promote the training of reception and archival professionals on archival techniques and methods and legislation that permeates patients' medical records; organize the patients' charts in the referenced health unit and implement a spreadsheet, facilitating the identification of patients' charts in a more agile way, thus helping the professionals' routine. Conclusion: From this study it was verified that the medical records are documents in which all the care carried out by the multiprofessional team is registered to each user. In this way, they are configured as important tools for: supporting scientific research in the health area, assisting professionals in monitoring and evaluating the health of their patients, planning health actions, analyzing the epidemiological profile of each territory, and serving as evidence for external demands, such as lawsuits, ethics and professional classes, accreditation, etc. Despite the above, it is noted that the treatment given to these documents is still far from ideal and the health unit in focus does not refute this reality, so the proposal presented here is necessary.

Descriptors: Medical Records; Inservice Training; Archives.

Resumen 
Objetivo: contribuir a la organización de los prontuarios en una unidad básica de salud (UBS) de Maceió. Método: para fundamentar el plan de intervención se realizaron investigaciones en la base de datos de la Scientific Electronic Library Online (SciELO), Google Académico con los descriptores: registros; capacitación en servicio y archivo. Los documentos de la Secretaría Municipal de Salud de Maceió y UBS también subsidiaron ese trabajo. Resultados esperados: promover la capacitación de los profesionales de la recepción y del archivo sobre técnicas y métodos archivistas y legislación que permea los prontuarios de los pacientes; organizar los prontuarios de los pacientes en la unidad de salud referenciada $e$ implantar una hoja de cálculo, facilitando la identificación de los prontuarios de los pacientes de manera más ágil, auxiliando así, la rutina de los profesionales. Conclusión: A partir de este estudio se verificó que los prontuarios son documentos en que se registra todos los cuidados realizados por el equipo multiprofesional a cada usuario. De este modo, se configuran como importantes herramientas para: subsidiar investigaciones cientificas en el área de la salud, auxiliar a los profesionales en el monitoreo $y$ evaluación de la salud de sus pacientes, planificación de las acciones de salud, análisis del perfil epidemiológico de cada territorio, además de servir de prueba cabal para demandas externas, como acciones judiciales, del consejo de ética y de clases profesionales, acreditación etc. A pesar de lo expuesto, se nota que el tratamiento dado a esos documentos aún está lejos de ser el ideal y la unidad de salud en foco no refuta esa realidad, luego la propuesta aqui presentada se hace necesaria.

\section{Descriptores: Registros Médicos;}

Capacitación en Servicio; Archivos.

\section{Introdução}

$\mathrm{O}$ atendimento aos usuários em uma unidade de saúde abrange vários profissionais, os quais podem ser médicos, enfermeiros, fisioterapeutas, terapeutas ocupacionais, nutricionistas, biomédicos, educadores físicos, odontólogos, assistentes sociais, fonoaudiólogos, psicólogos, dentre outros. Uma atividade de extrema relevância executada por esses profissionais é o registro de cada atendimento, ação que pode contribuir para análise da situação de saúde, 
fundamentar o histórico clínico do paciente e para atendimentos futuros. $\mathrm{O}$ registro das informações referentes ao atendimento a cada paciente normalmente é realizado por meio de $\operatorname{prontuários}^{(1)}$.

O Conselho Federal de Medicina (CFM), através da Resolução 1.638 de 2002 em seu Art. $1^{\circ}$, define o prontuário médico como:

Documento único constituído de um conjunto de informações, de sinais e de imagens registradas, geradas a partir de fatos, acontecimentos e situações sobre a saúde do paciente e a assistência a ele prestada, de caráter legal, sigiloso e científico, que possibilita a comunicação entre membros da equipe multiprofissional e a continuidade da assistência prestada ao indivíduo ${ }^{(2)}$.

A constituição brasileira estabelece que a saúde é um direito de todos e dever do Estado, logo deve ser garantida através do acesso universal e igualitário a ações e serviços. Cuidar da saúde é atribuição da União, dos Estados, do Distrito Federal e dos Municípios, com necessidade de divisão de responsabilidade e de trabalho cooperativo e integrado entre as diferentes esferas de governo, para organizar o Sistema Único de Saúde (SUS). Partindo dessa lógica, o prontuário do paciente é um documento fundamental para a assistência integral e continuada ao paciente, colaborativamente edificado diante de informações registradas pela equipe multiprofissional de saúde sobre os aspectos físicos, mentais e sociais do paciente $^{(3)}$.

Trata-se do mais relevante meio de interlocução entre os membros da equipe de uma unidade orientada à saúde da população. A prática de registro de informações a respeito dos atendimentos prestados vem sendo realizada, há muitos anos. Nessa perspectiva, esse documento é considerado como um dossiê, em que o conteúdo evidencia várias informações sobre um determinado paciente, suas características, suas queixas e o tratamento a ele prescrito pelos profissionais que o atenderam $^{(4)}$.

Assim sendo, os prontuários são avalizados como repositórios informacionais de fundamental relevância no atendimento à saúde da população, sendo importantes bases de informação sobre determinada patologia, seu histórico, desenvolvimento, prescrições, curas ou medidas paliativas, entre outros $\operatorname{aspectos}_{\operatorname{associados}}^{(4)}$.

Os prontuários dos pacientes são compostos por documentos produzidos a partir das anotações de diagnósticos, resultados de exames, procedimentos empregados durante o atendimento e 
tratamento dos pacientes. As informações inseridas nos prontuários auxiliarão na continuidade do tratamento, podendo ser consultados os procedimentos que incidiram positivamente nesse processo ${ }^{(4)}$.

O prontuário incorpora também outros valores, servindo de prova para contestações éticas no âmbito jurídico, entre outros. Em suma, o prontuário apresenta o histórico dos atendimentos aos usuários nos serviços de saúde, e necessita ser o principal registro dessas informações, compondo assim, a base de um sistema de informação de saúde. Neste documento, essencialmente devem constar informações mínimas necessárias que permitam a equipe identificar os riscos não somente individuais, como também os riscos coletivos. Portanto, dados de identificação da estrutura familiar, aspectos sociais e econômicos (escolaridade, ocupação, renda etc.) e condições de moradia (ambiente e saneamento) devem fazer parte de um prontuário familiar $^{(5)}$.

A Associação Brasileira de Saúde Coletiva (Abrasco) menciona que a partir das novas demandas do Sistema Único de Saúde (SUS), as informações em saúde alcançam um papel estratégico na gestão do sistema, uma vez que os trabalhadores que exercem suas funções nesta área executam atividades cada vez mais complexas em seu processo de trabalho, demandando, desta forma, um novo perfil profissional. Apesar deste fato, o trabalho neste campo caracteriza-se, geralmente, por pouca disponibilidade de formação específica e por poucas regulamentações a respeito, o que confere aqueles que aos atuam neste campo uma fragilidade no que se refere à constituição do seu perfil e identidade profissional $^{(6)}$.

A qualidade na gestão dessas informações pode impedir disfuncionamentos, que vão desde a troca de pacientes ao erro no preparo e administração de medicamentos, além de outras ações equivocadas. Tais falhas podem acarretar o agravamento do estado e saúde do usuário (retrocesso na recuperação e risco de morte), comprometendo o bem-estar dos profissionais e ecoando negativamente na sociedade ${ }^{(7)}$.

O Ministério da Saúde (MS) focando na gestão da qualidade oficializou em 2004 o Programa Nacional de Avaliação dos Serviços de Saúde (PNASS) como evolução do Programa Nacional de Avaliação dos Serviços Hospitalares (PNASH), lançado em 1998, por esse mesmo órgão. No que se refere à gestão de 
prontuários em particular, o PNASS contempla critérios inseridos em padrões de conformidade. Padrões esses que são divididos em três blocos: Bloco I (Gestão Organizacional) Bloco II (Apoio Técnico e Logístico) e Bloco III (Gestão da Atenção à Saúde). O terceiro critério relacionado à Gestão Organizacional apresenta itens diretamente ligados à gestão de $\operatorname{prontuários}^{(7)}$.

Os itens acima citados referem-se a: 1) sistematização dos registros de atendimentos, evolução e intercorrências nas fichas ou prontuários dos pacientes; 2) segurança do armazenamento e alocação dos prontuários, até mesmo os de arquivo morto; o bom estado de conservação, organização e acessibilidade, observando o fluxo dos prontuários e a garantia da confiabilidade das informações contidas neste documento; 3) verificação, por meio de documento, das ações da comissão de revisão de prontuários e/ou óbito, quando existir. Nota-se, assim, que o PNASS não faz exigências quanto a um protocolo a ser seguido, contudo, o serviço de saúde deve atender aos padrões e critérios estabelecido $^{(7)}$.

Para tanto, as práticas arquivistas são essenciais no tratamento, armazenamento e recuperação de documentos. Toda instituição pública que tenha um mínimo de organização de seu acervo, mostra a importância que este zelo tem, uma vez que, o arquivo faz parte de um conjunto estrutural e, por conseguinte social. Logo, a gestão documental quando aplicada, confere uma eficiência no funcionamento das instituições, tornando o acesso e recuperação da informação mais ágil. No âmbito da saúde, essa eficácia precisa ser ainda mais otimizada, uma vez que uma decisão tomada ou baseada em dados incorretos pode acarretar risco à vida humana ${ }^{(5)}$.

Apesar das evidências sobre a importância dos portuários, o tratamento informacional desses documentos ainda se encontra em estado noviço, já que, por muito tempo, tais instrumentos não foram valorizados, tanto no campo da saúde, como também noutras searas ${ }^{(8)}$.

Para que ocorra mudança neste cenário os trabalhadores que possuem cargo de nível médio e que exercem funções relacionadas a registros $\mathrm{e}$ informações em saúde no SUS necessitam ser credenciados para seleção, gerenciamento e avaliação da informação adequada visando à contribuição para o processo de tomada de decisão e para o desenvolvimento da pesquisa em saúde. A qualificação 
desses atores deve proporcionar condições favoráveis para facilitar a continuidade do tratamento ao paciente; admitir a investigação e a pesquisa científica; oferecer às instituições de saúde os dados necessários para a avaliação da qualidade da assistência, a eficiência do trabalho dos profissionais; e, sobretudo, subsidiar as atividades de planejamento das ações de saúde e a aplicação de recursos ${ }^{(6)}$.

Ao tratar as informações de saúde como fonte de conhecimento sobre 'a situação de saúde de uma população’ torna-se fundamental assegurar-se um processo de organização adequado. Para tornar isso possível, os profissionais que atuam nessa área necessitam de uma formação que contemple o processo de produção de informações para o sistema de informações em saúde, para a vigilância em saúde, para a gestão do cuidado e do sistema de saúde, bem como para os usuários e demais profissionais da saúde ${ }^{(6)}$.

Os autores defendem ainda que a precariedade de profissionais sem a formação adequada para desenvolverem as atividades de registros e informações em saúde é expressiva no em todo território nacional. Essas atividades normalmente são executadas de forma deficiente, conduzindo a 'nós críticos' no que se refere a qualidade e uso dos dados e das informações para o SUS. Portanto, a partir do reordenamento do sistema, torna-se crucial a conformação de novos perfis profissionais, que devem ser definidos em virtude do modelo assistencial, da organização e da composição tecnológica dos serviços ${ }^{(6)}$.

Atualmente, o acervo da unidade básica de saúde (USB) em evidência é composto de aproximadamente 130.000 prontuários, separados por um sistema de códigos (numeração); onde os mesmos são organizados em pasta em ordem crescente de numeração e armazenados em gavetas. Por se tratar de um documento sigiloso o manuseio é restrito aos servidores que laboram na recepção, no próprio arquivo, como também pelas enfermeiras ${ }^{(9)}$.

Nos últimos anos houve um acréscimo significativo no número de usuários atendidos na UBS citada no paragrafo anterior e esse crescimento desordenado resultou no acúmulo de prontuários, gerando transtornos tais como: extravio de alguns desses documentos, prontuários avulsos por paciente, além de dificuldades para conservação e integridade física dessas ficha ${ }^{(9)}$. Diante do exposto, torna-se evidente a importância deste projeto que visa a organização dos prontuários dos pacientes nessa USB, de maneira a 
facilitar o acesso e manuseio, preservação e integridade física, segurança das informações clínicas dos usuários, além de atenuar os erros relacionados a esses documentos.

\section{Método}

O projeto de intervenção apresentado surgiu a partir da observação dos profissionais de saúde responsáveis pelo acolhimento aos usuários que procuram os serviços da USB.

O acolhimento nessa unidade é realizado sempre pelos trabalhadores que ocupam a recepção, com cargo de assistente administrativo, onde uma de suas funções é a abertura de prontuários dos pacientes, e recorrentemente realizam também o arquivamento desses documentos. Acontece que o setor de arquivo da unidade possui apenas um profissional e quando este precisa se ausentar pelos mais variados motivos, aqueles que integram a recepção necessitam realizar também as tarefas deste servidor ${ }^{(9)}$.

Os 7 trabalhadores que laboram na recepção e 01 do arquivo deverão ser contemplados com uma capacitação orientada a métodos arquivistas e legislação que permeiam os prontuários. Para tal, será fundamental o apoio de profissionais de referência nesta área tanto da Secretaria Municipal de Saúde (SMS) como da Secretaria Estadual de Saúde (Sesau). Assim, a autora do projeto visando concretizar tal apoio deverá realizar visita técnica nesses órgãos. Para eficácia da ação a Direção da unidade básica de saúde terá que ser sensibilizada sobre a importância da liberação dos profissionais para participarem da ação educativa. No intuito de não prejudicar o serviço prestado, deverá haver um rodízio dos profissionais que vão participar do curso.

Concluída a etapa de capacitação, os servidores deverão iniciar a organização dos prontuários, seguindo os métodos apreendidos no curso.

Será necessário o apoio dos profissionais da área de Tecnologia da Informação (TI) da USB para edificar o modelo de planilha eletrônica, onde serão inseridos os dados dos prontuários. A inserção dos dados relacionados aos prontuários nessa ferramenta será realizada pelos profissionais da recepção e do arquivo, fato que vai subsidiar a identificação desses documentos de maneira mais ágil.

Após a implementação da proposta de intervenção, serão realizadas avaliações semestrais do 
plano de ação com envolvimento de toda equipe que atua na recepção e no arquivo, permitindo com isso $\mathrm{o}$ surgimento de novas ideias para o projeto. A avaliação será focada na redução de erros relacionados aos prontuários, além da opinião da equipe acima citada.
Para fundamentar o plano foram realizadas pesquisas na base de dados da Scientific Electronic Library Online (SciELO), Google Acadêmico com os descritores: prontuários, capacitação em serviço, arquivo. Os documentos da SMS e da USB em questão também subsidiaram esse trabalho.

\section{Quadro 1 - Resumo do Plano de Intervenção}

\begin{tabular}{|c|c|c|c|c|}
\hline NÓ CRÍTICO & $\begin{array}{l}\text { OPERAÇÃO } \\
\text { (PROJETO) }\end{array}$ & $\begin{array}{l}\text { CRONOGRAMA DE } \\
\text { EXECUÇÃO }\end{array}$ & $\begin{array}{c}\text { RECURSOS } \\
\text { NECESSÁRIOS }\end{array}$ & $\begin{array}{l}\text { RESULTADOS } \\
\text { ESPERADOS }\end{array}$ \\
\hline $\begin{array}{l}\text { Os profissionais } \\
\text { que laboram na } \\
\text { recepção e no } \\
\text { arquivo não } \\
\text { possuem } \\
\text { capacitação sobre } \\
\text { técnicas e } \\
\text { métodos } \\
\text { arquivistas e } \\
\text { legislação que } \\
\text { permeia os } \\
\text { prontuários }\end{array}$ & $\begin{array}{l}\text { Capacitação dos } \\
\text { trabalhadores } \\
\text { Proporcionar } \\
\text { capacitação para } \\
\text { os trabalhadores }\end{array}$ & Encontros quinzenais & $\begin{array}{c}\text { *Motivação dos } \\
\text { profissionais; } \\
\text { *Sensibilização da } \\
\text { Direção da unidade; } \\
\text { *Apoio de } \\
\text { profissionais de } \\
\text { referência em arquivo } \\
\text { da SMS de Maceió } \\
\text { *Apoio da Sesau. }\end{array}$ & $\begin{array}{l}\text { *Profissionais } \\
\text { capacitados quanto } \\
\text { ao preenchimento } \\
\text { eficaz dos } \\
\text { prontuários e seu } \\
\text { armazenamento. } \\
\text { *Profissionais } \\
\text { munidos de } \\
\text { conhecimentos sobre } \\
\text { a legislação que } \\
\text { permeia o prontuário. }\end{array}$ \\
\hline $\begin{array}{l}\text { Os prontuários da } \\
\text { unidade não estão } \\
\text { organizados por } \\
\text { meio de técnicas } \\
\text { arquivistas }\end{array}$ & $\begin{array}{l}\text { Organização dos } \\
\text { prontuários } \\
\text { Efetivar a } \\
\text { Organização dos } \\
\text { prontuários da } \\
\text { unidade por meio } \\
\text { de técnicas } \\
\text { arquivistas }\end{array}$ & & $\begin{array}{l}\text { *Motivação dos } \\
\text { profissionais } \\
\text { *Aquisição de } \\
\text { material de } \\
\text { expediente. }\end{array}$ & $\begin{array}{l}\text { *Prontuários da } \\
\text { unidade organizados. } \\
\text { *Diminuição de } \\
\text { erros relacionados ao } \\
\text { arquivamento de } \\
\text { prontuários; } \\
\text { *Fortalecimento do } \\
\text { processo de trabalho } \\
\text { dos setores de } \\
\text { recepção e arquivo. }\end{array}$ \\
\hline $\begin{array}{l}\text { Existência de } \\
\text { prontuários } \\
\text { avulsos }\end{array}$ & $\begin{array}{l}\text { Implantação de } \\
\text { uma planilha } \\
\text { eletrônica } \\
\text { Implantar uma } \\
\text { planilha eletrônica } \\
\text { contendo } \\
\text { informações dos } \\
\text { prontuários para } \\
\text { subsidiar a } \\
\text { identificação } \\
\text { desses documentos } \\
\text { de maneira mais } \\
\text { ágil, descartando } \\
\text { assim a } \\
\text { possibilidade da } \\
\text { edificação de } \\
\text { prontuários } \\
\text { avulsos. }\end{array}$ & & $\begin{array}{c}\text { Motivação dos } \\
\text { profissionais; } \\
\text { Auxilio dos } \\
\text { profissionais de TI } \\
\text { para edificar o modelo } \\
\text { da planilha. } \\
\text { Inserção dos dados } \\
\text { dos prontuários na } \\
\text { planilha pelos } \\
\text { profissionais da } \\
\text { recepção e arquivo. }\end{array}$ & $\begin{array}{c}\text { *Agilidade na } \\
\text { identificação dos } \\
\text { prontuários. } \\
\text { *Eliminação de } \\
\text { prontuários avulsos; }\end{array}$ \\
\hline
\end{tabular}




\section{Resultados Esperados}

Pretende-se que esse projeto possa contribuir para a organização dos prontuários na USB, adotando como estratégia a capacitação dos profissionais do arquivo e recepção em técnicas arquivistas e legislação condizente a esses documentos, como também a implantação de uma planilha eletrônica, com a finalidade de identificar os prontuários de maneira dinâmica, evitando assim, a abertura de novos registros a usuários já cadastrados.

\section{Conclusão}

Notou-se que no âmbito nacional o tratamento dado aos prontuários ainda está longe de ser o ideal e USB aqui apresentada não refuta essa realidade. $\mathrm{Na}$ unidade referenciada, o aumento desenfreado no número de atendimentos vem proporcionando acúmulo de prontuários, sendo esses armazenados de forma inadequada, ocasionando diversos problemas para o serviço. Logo, espera-se que a intervenção possa proporcionar mudanças significativas neste contexto, ocasionando satisfação dos profissionais e usuários vinculados a USB.

\section{Referências}

1. Almeida MB, Andrade, A.Q. Organização da informação em prontuários de pacientes: uma abordagem Popperiana. Informação \& Tecnologia (João Pessoa) [periódico na internet]. 2014 [citado 2016 jul. 01]; 1(1): 29-41. Disponível em: http://periodicos.ufpb.br/ojs/index.php/ itec/article/view/19195.

2. Conselho Federal de Medicina (BR). Resolução 1638 de 09 de agosto de 2002: define prontuário médico e torna obrigatória a criação da comissão de prontuário nas instituições de saúde. Diário Oficial da União; Poder Executivo, n. 153, Seção 1, 184-5. Brasília: Conselho Federal de Medicina; 2002.

3. Galvão MCB, Ricarte ILM. O prontuário eletrônico do paciente no século xxi: contribuições necessárias da ciência da informação. InCID: Revista de Ciência da Informação e Documentação. Ciência da Informação (São Paulo) [periódico na internet]. 2011 [citado 2016 ago. 22]; 2(2): 7700. Disponível em: www.revistas.u sp.br/incid/article/view/42353/46024.

4. Lunardelli, RSA, Tonello IMS, Molina LG. A constituição da memória dos procedimentos em saúde no contexto do prontuário eletrônico do paciente. Informação \& Informação. (Londrina) [periódico na internet]. 2014 [citado 2016 ago. 22]; 19(3): 107-24. Disponível em: http://www. uel.br/revistas/uel/index.php/informaca o/article/view/18837.

5. Leite JR, Souza ACM. Práticas arquivistas no contexto de prontuários médicos: um estudo em unidade de saúde da família. Paraíba: Archeion Online; 2016. 3(2): 55-4. 
6. Morosini MVGC, Lopes MCR, Chagas DC, Chinelli F, Vieira M, organizadores. Trabalhadores técnicos em saúde: aspectos da qualificação profissional no sus. Rio de Janeiro: Escola Politécnica de Saúde Joaquim Venâncio; 2013: 179-05.

7. Aquino PLS. Segurança do paciente na gestão de prontuários. In: Pinho DLM, Abrahão JL, Ferreira MC. As estratégias operatórias e a gestão da informação no trabalho de enfermagem no contexto hospitalar. Paraíba: Universidade Federal da Paraíba; 2015.

8. Pinto VB, Farias KM, Meneses BC. Epistemologia do registro e da organização do conhecimento no contexto da saúde: o caso do registro do paciente. Ciências da Informação (Ceará) [periódico na internet]. 2012 [citado 2016 ago. 24]; 19(3): 455-70. Disponível em: http://www.iskoib erico.org/wp-content/uploads/2014/09/ 455-470_Bentes-Pinto.pdf.

9. Secretaria Municipal de Saúde de Maceió (AL). Unidade Básica de Saúde de Maceió. Relatório Técnico. Alagoas: Secretaria Municipal de Saúde; 2016. 